\title{
APPLICATION OF THE GALERKIN METHOD TO PROBLEMS IN HYDRODYNAMIC STABILITY*
}

\author{
BY \\ RICHARD C. DI PRIMA** \\ Massachusetts Institute of Technology
}

1. Introduction. Problems in hydrodynamic stability fall essentially into two categories: those in which the instability may be traced to the action of viscosity and those in which the instability may be attributed to some other factor such as centrifugal force. Examples of the former are the nearly parallel, viscous flows, where it is sufficient to consider only two dimensional disturbances. In the latter class are the problems of flow between rotating, concentric cylinders and over concave walls; here three dimensional disturbances must be considered. In these problems there will be no "inner friction layer" and hence there should be no rapid oscillation of the eigenfunction. Consequently such problems should be amenable to treatment by the Galerkin method.

The stability of a viscous fluid contained between two concentric rotating cylinders was first investigated experimentally and analytically by G. I. Taylor [1]. When the cylinders rotate in the same direction investigations have also been carried out by Jeffreys [2], Synge [3], Pellew and Southwell [4] and Meksyn [5]. If the cylinders rotate in opposite directions, more formidable mathematical problems are presented: however the work of G. I. Taylor still applies, though the calculations become much more tedious. Also, Meksyn [6, 7] has obtained asymptotic representations of the stability equation in this case.

In Sec. 2, this problem is formulated in a slightly different manner from that adopted by Taylor and Meksyn. This formulation brings out clearly the structure of the eigenvalue as well as its dependence on two other parameters. Explicitly, $S$, the eigenvalue defined in Sec. 2 , is a function of $\lambda d_{1}$ and $d_{1} / d$. Here $2 \pi / \lambda$ is the wave length in the axial direction, $d$ is the distance between the cylinders and $d_{1}$ is the distance from the inner cylinder to the vanishing point of the mean velocity. It is shown, with the help of experimental data, that for small $d_{1} / d, S=S\left(\lambda d_{1}, 0\right)$ and for $d_{1} / d$ greater than roughly .55 the dependence of $S$ on $d_{1} / d$ cannot be neglected. The Galerkin method is used to solve the resultant eigenvalue problem. Computations are carried out for $d_{1} / d$ small, and $d_{1} / d=1$, obtaining results in agreement with the experimental data given by Taylor (see Figs. 1 and 2). The result for small $d_{1} / d$ agrees with that obtained by Meksyn. In the transition range from $\lambda d_{1}=$ constant to $\lambda d=\pi$ (obtained when the cylinders rotate in the same direction, $d_{1} / d>1$ ) where the dependence on $d_{1} / d$ may not be neglected, the method is directly applicable though the calculations will be slightly more lengthy.

The stability of a viscous flow over a concave surface was first investigated by Görtler [8] and later by Meksyn [9] who obtained different results. Because of the discrepancy in the results as well as to see if the Galerkin method might be applicable in

*Received March 25, 1954. This work was sponsored by the Office of Naval Research under Contract N 5 ori- 07871.

**The author wishes to thank Professor C. C. Lin for suggesting this investigation and also for his many helpful discussions during the course of the work. Thanks are also due to the Aeroelastic Computing Laboratory and Miss Ethel Ginsburgh who carried out the computations. 


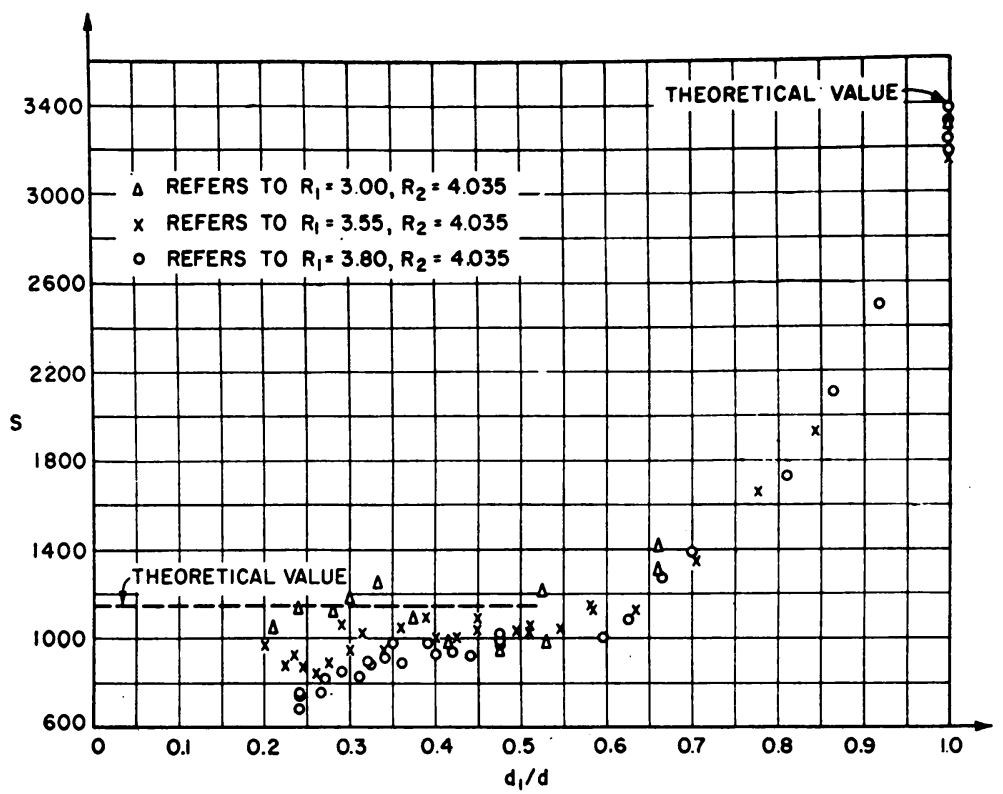

Fig. 1. $S$ (Experimental) vs. $d_{1} / d$.

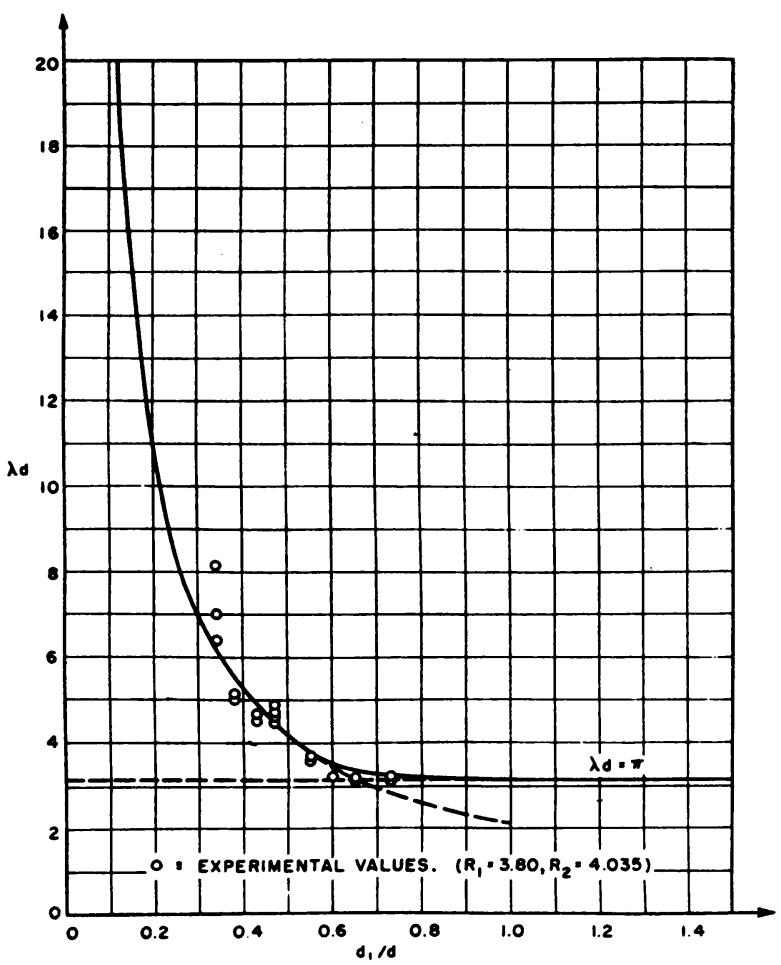

Fra. 2. $\lambda d$ ve. $d_{1} / d$. 
a future study of the stability of a compressible flow over curved plates, some rough computations were carried out for this problem. The results obtained in Sec. 3 tend to confirm those of Görtler.

2. Rotating cylinders. For a viscous incompressible flow between two coaxial, infinitely long cylinders of radii $R_{1}$ and $R_{2},\left(R_{2}>R_{1}\right)$ and angular velocities $\Omega_{1}$ and $\Omega_{2}$ the equations of motion admit the stationary solution

$$
V=A r+\frac{B}{r}
$$

The constants $A$ and $B$, determined by the boundary conditions, are

$$
A=\frac{\Omega_{1}\left(1-\mu R_{2}^{2} / R_{1}^{2}\right)}{1-R_{2}^{2} / R_{1}^{2}}, \quad B=\frac{\Omega_{1}(1-\mu) R_{1}^{2}}{1-R_{1}^{2} / R_{2}^{2}}
$$

where $\mu=\Omega_{2} / \Omega_{1}$.

Superimposing on this basic flow a symmetrical three dimensional disturbance we obtain the following linearized differential equation for marginal stability:*

$$
\left(D D^{*}-\lambda^{2}\right)^{3} v=\frac{4 A \lambda^{2}}{\nu^{2}}\left(A+\frac{B}{r^{2}}\right) v .
$$

Here the wave length of the disturbance in the axial direction is $2 \pi / \lambda, \nu$ is the kinematic viscosity, $D=d / d r, D^{*}=D+1 / r$, and $v$ is the radial component of the disturbance. The boundary conditions are

$$
v=0, \quad\left(D D^{*}-\lambda^{2}\right) v=0, \quad D^{*}\left(D D^{*}-\lambda^{2}\right) v=0,
$$

at $r=R_{1}$ and $r=R_{2}$.

In accordance with the work of previous investigators it is assumed that the difference in the radii of the two cylinders is small compared with their mean, i.e.

$$
d=\left(R_{2}-R_{1}\right) \ll \frac{1}{2}\left(R_{1}+R_{2}\right) .
$$

In this case $d$ is the important scale in the motion, $D=0(1 / d), \lambda=0(1 / d)$ and $1 / r$ may be neglected compared with $D$. Consequently Eqs. (2.3) and (2.4) reduce to the following equations given by Jeffreys [2]

$$
\left(D^{2}-\lambda^{2}\right)^{3} v=\frac{4 A \lambda^{2}}{\nu^{2}}\left(A+\frac{B}{r^{2}}\right) v
$$

and

$$
v=0, \quad\left(D^{2}-\lambda^{2}\right) v=0, \quad D\left(D^{2}-\lambda^{2}\right) v=0,
$$

at $r=R_{1}$ and $r=R_{2}$.

When the cylinders rotate in the same direction the basic angular velocity, $A+B / r^{2}$, may be approximated by a constant. The resultant eigenvalue problem requires the minimization of a critical parameter $T\left(\Omega_{1}, \mu, R_{1}, R_{2}\right)$ with respect to $\lambda d[4]$. This minimum occurs approximately when $\lambda d=\pi$, a result which will be used later.

For the problem under consideration, in which the cylinders rotate in opposite directions, $A+B / r^{2}$ is approximated by a straight line profile which has the slope of

*Eliminate $u$ from Eqs. (51) and (54) of [10] after setting $H$ and $\psi=0$. 
$V / r$ at $R_{0}, R_{0}$ being determined by $V\left(R_{0}\right)=0$. It is easy to show that

$$
R_{0}=\frac{(1-\mu)^{1 / 2}}{\left(1-\mu R_{2}^{2} / R_{1}^{2}\right)^{1 / 2}} .
$$

Setting $r=R_{0}+\zeta$ we obtain, upon dropping terms of order $\left(\zeta / R_{0}\right)^{2}$, the relation

$$
A+\frac{B}{r^{2}} \cong \frac{2 A}{R_{0}} \zeta \text {. }
$$

Let

$$
x=\frac{1}{d_{1}}\left(d_{1}+\zeta\right), \quad a=\lambda d_{1}, \quad S=\frac{8 A^{2} d_{1}^{5}}{\nu^{2} R_{0}}=\frac{8 \Omega_{1}^{2}\left(1-\mu R_{2}^{2} / R_{1}^{2}\right) d_{1}^{5}}{\nu^{2}\left(1-R_{2}^{2} / R_{1}^{2}\right) R_{0}},
$$

where $d_{1}=R_{0}-R_{1}$; then from Eqs. (2.6) and (2.7)

$$
\left(D^{2}-a^{2}\right)^{3} v=S a^{2}(x-1) v
$$

and

$$
v=0, \quad\left(D^{2}-a^{2}\right) v=0, \quad D\left(D^{2}-a^{2}\right) v=0,
$$

at $x=0$ and $x=d / d_{1}>1$, where $D=d / d x$.

The stability problem requires, for given values of the physical parameters $R_{1}$, $R_{2}, \mu$, the determination of the minimum value of $\Omega_{1}$ with respect to $a$. From the form of $S$ we see that minimizing $\Omega_{1}$ for given $R_{1}, R_{2}$, and $\mu$ means minimizing $S$. It is clear from Eqs. (2.11) and (2.12) that $S$ is a function of $a=\lambda d_{1}$, and also $d / d_{1}$. Hence for each new set of physical constants $R_{1}, R_{2}, \mu$ the minimization with respect to $a$ must be carried out anew. Fortunately, however, it is possible for a wide range of values of $d / d_{1}$ to neglect the dependence of $S$ on $d_{1} / d$ and then the minimization with respect to $a$ may be carried out once and for all.

The possibility of neglecting the dependence of $S$ on $d_{1} / d$ is motivated by the fact that the centrifugal force will tend to cause instability in the region $R_{1}<r<R_{0}$ and will tend to stabilize the flow in the region $R_{0}<r<R_{2}{ }^{*}$ Now if $R_{0}$ is close to the inner cylinder, the exact position of the outer cylinder should have very little effect on the eigenvalue problem as long as it is sufficiently far away from $R_{0}$ and hence we can let $R_{2} \rightarrow \infty$. That is, for our eigenvalue problem, if $d / d_{1}>1 / \delta>1$ where $\delta$ is sufficiently small we may set $d_{1} / d=\infty$ in the boundary conditions (2.12). To check the validity of this argument and also to estimate the size of $\delta$, values of $S$ were computed using the experimental data of Taylor and plotted against $d_{1} / d$. The results are exhibited in Fig. 1 . For $\delta$ somewhere between .5 and .6 we see from Fig. 1 that for $d_{1} / d<\delta$, there is very little variation of $S$ with $d_{1} / d$ and the above argument is verified.

It should be pointed out here that there is possibly an error in the data for $R_{1}=3.80$ ([6], p. 491) and probably those points should be slightly higher.

The analysis, using a linear profile for $V / r$, may be summarized in the following manner. For $0<d_{1} / d<\delta, S=S\left(\lambda d_{1}, 0\right)$; for $\delta<d_{1} / d<1, S=S\left(\lambda d_{1}, d_{1} / d\right)$, and for $d_{1} / d>1, T=T(\lambda d)$. Then $\delta<d_{1} / d<1$ is the transition range from $\lambda d_{1}=$ constant to $\lambda d=$ constant and in that range the dependence on $d_{1} / d$ cannot be neglected.

First we treat the case in which $0<d_{1} / d<\delta$, and the outer boundary conditions are to be applied at $\infty$. Then the eigenvalue problem involves only the three boundary conditions at $x=0$ since we seek solutions which die out exponentially at $\infty$. This

*See ([1], p. 327) and [2] for a more detailed discussion. 
problem is equivalent to that formulated by Meksyn [6]. Formal application of the Galerkin method yields the eigenvalue determinant

$$
\left|I_{i i}-S a^{2} J_{i i}\right|=0
$$

where

$$
I_{i i}=\int_{0}^{\infty} v_{i}\left(D^{2}-a^{2}\right)^{3} v_{i} d x, \quad J_{i i}=\int_{0}^{\infty}(x-1) v_{i} v_{i} d x .
$$

The functions $v_{i}$ are taken to be of the form $e^{-m x}\left[x^{3}+b\left(m x^{2}+x\right)\right]$ and $e^{-m x} x^{n}$ for $n \geq 4$, where $b$ is determined by the boundary conditions (2.12).

Because of the change in sign of the term $x-1$ in Eq. (2.11) some difficulty in applying the Galerkin method is to be expected. In order to approximate the correct mode, i.e. the mode corresponding to the smallest positive eigenvalue, using only a few functions it is necessary to make several preliminary considerations. First from Eq. (2.11) we have for $x$ large that $v$ will behave roughly as $\exp \left[-\left(S a^{2}\right)^{1 / 6} x^{7 / 6}\right]$. Reasonable values of $S$ and $a$ from the work of Taylor and Meksyn are 1000 and 2; hence likely values for $m$ are 4,5 , or 6 . Also taking $v_{i}=e^{-m x} x^{n}$ for $n \geq 4$ it is easy to show that $I_{i i}<0$, and $J_{i i} \lesseqgtr 0$ as $m$ is Thus for any single function to give a positive eigenvalue it is necessary that its maximum be inside of $1-1 / 2 m$. This is in accord with the remarks made in the preceding paragraphs.

If all combinations of two functions having maxima within this critical value gave satisfactory results, it should be expected that three such functions would give an accurate value for $S$, even though extraneous roots might be introduced. For $a=2$ the

\begin{tabular}{|c|c|}
\hline Function & Has maximum at $x=$ \\
\hline$v_{1}=e^{-5 x}\left[x^{3}+b_{1}\left(5 x^{2}+x\right)\right]$ & 0.43 \\
\hline$v_{2}=e^{-4 x}\left[x^{3}+b_{2}\left(4 x^{2}+x\right)\right]$ & 0.53 \\
\hline$v_{3}=e^{-5 x} x^{4}$ & 0.8 \\
\hline$v_{4}=e^{-4.5} x^{4}$ & 0.89 \\
\hline$v_{5}=e^{-3 x} x^{4}$ & 1.0 \\
\hline
\end{tabular}
following table was constructed

Two term approximations using various combinations of these $v_{i}$ gave the following results: $S_{13}=876, S_{23}=968, S_{24}=1107$ where the subscripts refer to the functions used. Since the results of these calculations were satisfactory, a three term approximation for $v$ was used and the minimum value of $S$ with respect to $a$ was determined graphically. The final result was

$$
S_{123}=1075, \quad a=2.125 .
$$

Meksyn gives $S=1132, a=2$; the mean percentage difference in $S$ is 5 per cent. To investigate the effect of using an undesirable function (if only a few are to be used) the function $v_{2}$ was replaced by $v_{5}$ with the result that $S_{135}=1200$ at $a=2.25$. The percentage difference based on $S_{123}$ is 11.5 per cent which though not good is acceptable. 
Both $S_{123}$ and the Meksyn results are in agreement with the experimental values of $S$ if the data for $R_{1}=3.80$, which is probably in error, is not considered.

For $\delta<d_{1} / d<1$ the limiting case when $d_{1} / d=1$ was also computed. In this case the function $(x-1)$ does not change sign and no difficulty is encountered in using the Galerkin method. Analogously to the work of Pellew and Southwell [4], the function $P$ is introduced by

$$
P=\left(D^{2}-a^{2}\right) v .
$$

The boundary conditions (2.12) are then $P=0, D P=0, v=0$ at $x=0$ and $x=1$. Now, $P$ is expanded in a series of functions $P_{i}$ satisfying $P_{i}=D P_{i}=0$ at $x=0$ and $x=1$, and the $v_{i}$ are obtained by integrating Eq. (2.15) subject to $v=0$ at $x=0$, and $x=1$. (Introduction of the function $P$ simplifies considerably the computational work. It was not used in the previous case because it was desirable there to have approximations for $v$ which did not depend on $a$.) Using the $P_{i}$ as the weighting functions, we obtain the eigenvalue determinant

$$
\left|I_{i i}^{\prime}-S a^{2} J_{i i}^{\prime}\right|=0
$$

where

$$
\begin{aligned}
& I_{i j}^{\prime}=\int_{0}^{1}\left\{\left(D^{2}-a^{2}\right) P_{i} \cdot\left(D^{2}-a^{2}\right) P_{i}\right\} d x, \\
& J_{i i}=-\int_{0}^{1}(x-1)\left\{D y_{i} D y_{i}+a^{2} y_{i} y_{i}\right\} d x+\int_{0}^{1}\left(D y_{i}\right) \cdot y_{i} d x .
\end{aligned}
$$

The functions $P_{i}$ are the following: $P_{1}=x^{2}(x-1)^{2}$ which is symmetric about $x=1 / 2$, with maximum there, and $P_{2}=x^{2}(x-1)^{3}$ with minimum at $x=2 / 5$. Carrying out the computations we obtain

$$
S=3390, \quad a=\lambda d=3.15, \quad \text { for } \quad d_{1} / d=1 .
$$

This result is within 4.5 per cent of the average value of the experimental data, also $a=3.15$ is in agreement with the value of $\lambda d$ obtained by approximating the velocity profile by a constant, when the cylinders rotate in the same direction.

Using this result and the fact that $\lambda d=2.125 d / d_{1}$ for $0<d_{1} / d<\delta$ it is possible to draw a smooth curve for $\lambda d v s$. $d_{1} / d$ which gives the value of $\lambda d$ for the interval $\delta<d_{1} / d<1$. This curve is shown in Fig. 2. The dotted curve is the extension of $\lambda d_{1}=2.125$ past the range of validity of that result, roughly $\delta=.55$, as observed from Fig. 1. The circles indicate the experimental results of Taylor.

Finally, the method just discussed in which the function $P$ is approximated should apply readily for values of $d_{1} / d$ less than one, but still close to one. That is, as long as the function $x-1$ is negative over the major part of the range. A reasonable lower bound for $d_{1} / d$ might be .75 . It may be necessary to take an additional term in the approximation.

3. Flow over concave surfaces. The differential equations and boundary conditions governing marginal stability of three dimensional disturbances for an incompressible, viscous flow over a curved surface are

$$
\begin{gathered}
\left(D^{2}-\sigma^{2}\right) u-(d U / d \eta) v=0 \\
\sigma^{2} \mu U u+\left(D^{2}-\sigma^{2}\right)^{2} v=0
\end{gathered}
$$


and

$$
u=0, \quad v=0, \quad D v=0,
$$

at $\eta=0$, and $\eta=\infty$ ([8] Eq. (2.7)). In this derivation it was assumed that the component of the basic flow perpendicular to the wall was identically zero. Also the disturbances were assumed to be independent of $x$, the arc length along the plate, and of wave length $\lambda$ in the $z$ direction parallel to the plate. In Eqs. (3.1), $U$ is the basic flow; $\delta$ is the length scale in the $y$ direction; $\eta=y / \delta ; \sigma=\alpha \delta$ where $\alpha=2 \pi / \lambda ; \mu=2\left(U_{0} \delta / \nu\right)^{2}$ $(\delta / r) ; r$ is the constant radius of curvature, taken positive for the concave side of the surface; and $D=d / d \eta$.

Görtler [8] solves the system of equations (3.1) by using Green's functions to convert them to integral equations, which he then solves approximately. Meksyn [9] eliminates $v$ from Eqs. (3.1) and then obtains an asymptotic solution in terms of the large parameters $\sigma$ and $\mu$. Actually $\sigma=0(1)$; this may explain the discrepancy between Meksyn's results and the work of Görtler mentioned in the introduction.

It is clear from Eqs. (3.1) that the difficulties discussed in the previous section will not be present here. However, to use the Galerkin method, it is most convenient to retain Eqs. (3.1) as they are and approximate both $u$ and $v$. Then an $n$ term approximation for each of the velocities requires the solution of an eigenvalue determinant of the order of $2 n$, and hence the computational work is more than doubled for each succeeding term in the approximations for $u$ and $v$.

The basic velocity $U$ is approximated by the straight line profile, $U=\eta$ for $0 \leq \eta \leq 1$, $U=1$ for $\eta \geq 1$. This was the case considered by Meksyn and as one particular example by Görtler. The disturbance velocities $u$ and $v$ are approximated by the simplest functions satisfying the appropriate boundary conditions:

$$
\begin{aligned}
& u=e^{-a \eta}\left(A_{1} \eta+A_{2} \eta^{2}+\cdots\right), \\
& v=e^{-b \eta}\left(B_{1} \eta+B_{2} \eta^{2}+\cdots\right) .
\end{aligned}
$$

The choice of $a$ and $b$ depend upon the number of terms of the series which are to be taken. One wants to insure that each individual term dies out sufficiently rapidly as $\eta \rightarrow \infty$, but also the lower order terms should not die out too rapidly. In this work .only two terms of each series are used, as primarily the interest was of a qualitative nature. The constants were taken to be $a=b=1$.

Applying the Galerkin method in the usual manner the neutral curve given in Fig. 3

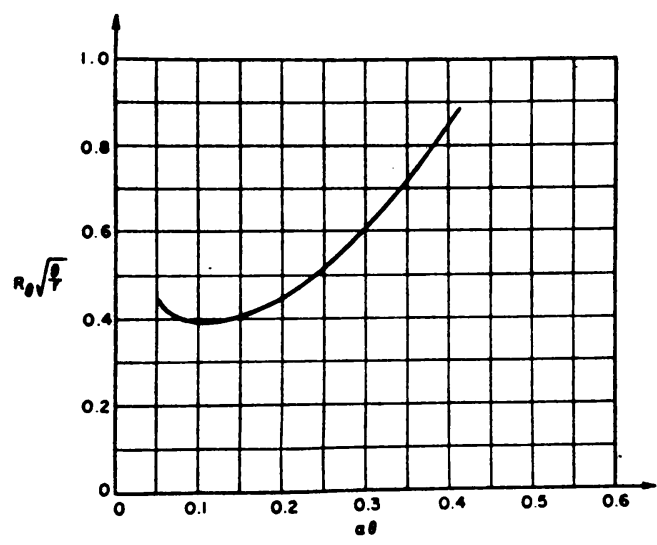

Fig. 3. 
was obtained. The results are expressed in terms of the momentum thickness $\theta([9]$, p. 261), $R_{0}=U_{0} \theta / \nu$. The critical value obtained by Görtler for this case was $R_{\theta}(\theta / r)^{1 / 2}=.83$ at $\alpha \theta=.15$, by Meksyn $R_{\theta}(\theta / r)^{1 / 2}=3.65$ at $\alpha \theta=.400$, and by this author $R_{\theta}(\theta / r)^{1 / 2}=.39$ at $\alpha \theta=.1$. This result* then confirms the magnitude of the critical parameter given by Görtler. In performing the present calculations, a second critical value of $R_{0}(\theta / r)^{1 / 2}$ was obtained for the higher mode. It is interesting to note in passing that this value is of the order of 2, i.e., comparable with Meksyn's value.

\section{Biblography}

1. G. I. Taylor, On the stability of a viscous liquid contained between two rotating cylinders, Phil. Trans. A223, 289-343 (1923)

2. H. Jeffreys, Some cases of instability in fluid motion, Proc. Roy. Soc. A118, 195-208 (1928)

3. J. L. Synge, On the stability of a viscous liquid between rotating cylinders, Proc. Roy. Soc. A167, 250-256. (1938)

4. A. Pellew and R. V. Southwell, On maintained convective motion in a fluid heated from below, Proc. Roy. Soc. A176, 312-344 (1940)

5. D. Meksyn, Stability of viscous flow between rotating cylinders I, Proc. Roy. Soc. A187, 115-128 (1946)

6. D. Meksyn, Stability of viscous flow between rotating cylinders II, Proc. Roy. Soc. A187, 480-491 (1946)

7. D. Meksyn, Stability of viscous flow between rotating cylinders III, Proc. Roy. Soc. A187, 492-504 (1946).

8. H. Görtler, Uber eine dreidimensionale Instabilitat laminarer Grenzschichten an konkaven Wanden, Nachr. Ges. Wiss. Gottingen, 1-26 (1940)

9. D. Meksyn, Stability of viscous flow over concave cylindrical surfaces, Proc. Roy. Soc. A203, 253-265. (1950)

10. S. Chandrasekhar, The stability of viscous flow between rotating cylinders in the presence of a magnetic. field, Proc. Roy. Soc. A216, 293-309 (1953)

*After this work was completed, the author received a report by Mr. A. M. O. Smith, On the Growth. of Taylor-Görtler vortices along highly concave walls, Douglas Aircraft Report No. ES 17110. He makes a rather complete investigation of this problem for the Blasius profile, using the Galerkin method with different choices of the constants $a$ and $b$ and six term approximations. His result for the critical parameter is about 2/3 that obtained by Görtler, which again tends to confirm the conclusion given in this paper. It should also be mentioned that his equations are more accurate than those used by Görtler. 